\title{
Research on the Overall Function Framework Scheme of the Intelligent Connected Vehicle
}

\author{
Xu Chao \\ Department of Mechanical Engineering, Shandong University, Jinan, China
}

\section{Email address:}

chaoxu2009@126.com

\section{To cite this article:}

Xu Chao. Research on the Overall Function Framework Scheme of the Intelligent Connected Vehicle. Science Discovery.

Vol. 5, No. 2, 2017, pp. 94-99. doi: 10.11648/j.sd.20170502.13

Received: March 31, 2017; Accepted: April 17, 2017; Published: April 20, 2017

\begin{abstract}
The Intelligent connected vehicle is car which is able to access the Internet, smart devices, other vehicles, and the road infrastructure. In the meantime, the intelligent connected vehicle can collect real-time data from multiple sources, and play a fundamental role in the future of the Internet of things. In the background of increasingly fierce competition in the industry, a large number of car manufacturers, software developers and hardware developers are doing their best to provide the best solution for the Intelligent connected vehicles. The Intelligent connected vehicles are expected to liberate drivers from tense and heavy driving operation, and to provide them with driving AIDS and entertainment functions. It is discussed in this paper that the possibility of the intelligent connected vehicle and relevant issues, and the hardware, and software solutions, their main characteristics are listed.
\end{abstract}

Keywords: The Intelligent Connected Vehicle, Vehicular Service, Vehicular Communication, Automatic Driving

\section{关于智能网联汽车的整体功能构架方案的研究}

\section{晁旭}

机械学院, 山东大学, 济南, 中国

邮箱

chaoxu2009@126.com

摘要: 智能网联汽车, 是一种能够接入互联网, 与智能设备、其他汽车、道路基础设施通信的汽车。同时, 智能网联 汽车能从多个来源收集实时数据, 在将来的物联网中发挥基础性作用。在行业竞争日趋激烈的大背景下, 大量的汽车 制造商、软件开发商、硬件开发商正在为智能网联汽车提供最佳的解决方案。智能网联汽车期望将驾驶员从紧张而繁 重的驾驶操作中解放出来, 为他们提供驾驶辅助及娱乐功能。本文论述了汽车智能网联实现的可能性及相关问题, 并 给出了一种解决方案。

关键词: 智能网联汽车, 汽车服务, 车载通信, 自动驾驶

\section{1. 引言}

在世界上第一辆汽车诞生后的一个多世纪中, 伴随着 科学技术的进步, 汽车的功能和性能不断革新。20世纪 80
年代, 汽车第一次引入了软件组件, 此后汽车的服务功能 呈现出体系化的发展态势。到 20 世纪 90 年代, 延长802.11 无线标准的协议的出现使得路上的不同车辆之间, 以及汽 车与智能道路基础设施之间能够通信。近些年, 汽车已经 实现了与网络之间的通信, 借鉴智能手机的方式, 将功能 
整合到汽车仪表板上, 为驾驶员和乘客提供先进的驾驶辅 助、多媒体娱乐服务。这种车载仪表板的联网功能设备是 智能网联汽车的必要元素, 随着智能手机和可穿戴设备的 发展, 汽车与它们之间的联系也愈加重要。因此网联车被 视为 “为驾驶员和乘客所使用的所有移动设备提供互联网 接口的汽车”, 也正如IBM在世界汽车新闻大会上所说, 网联车是 “能够与多个系统无缝连接的汽车, 是将消费者 连接到数字世界的汽车”。

在本文中, 定义智能网联车 [1]如下:

(1) 配备了一套智能化的应用服务, 具备动态语境功 能, 为驾驶员和乘客提供先进的辅助和娱乐功能;

(2) 能够与其他车辆互动, 促进车车之间的通信技术 发展, 能够与路上的其他智能设备沟通, 促进车路基础设 施间的通信技术发展;

(3) 具有自主驾驶功能。

本文的给出了一个智能网联汽车的解决方案, 针对上 文中定义的三个特征, 共分四部分讲述。在第二节中, 给 出了智能网联汽车提供的主要服务。为了更好地支撑服务 功能, 在第三节中, 继续讨论了所需收集的汽车数据, 以 及涉及的法律问题; 在第四节中, 给出了智能网联汽车所 需的通信技术; 在第五节中, 讨论自动驾驶汽车的可能性 和安全性。

\section{2. 汽车服务}

当前, 汽车用户对驾驶舒适度和智能度的要求越来越 高, 现代汽车除了必须提供所有传统汽车的服务外, 还需 要提供更多的基于汽车仪表板（如图1所示）的功能来满 足用户的需要, 如驾驶安全辅助、智能导航、能源优化、 智能娱乐、车辆共享等。本文基于此, 对于未来的智能网 联汽车提出了几种主要的辅助驾驶服务:

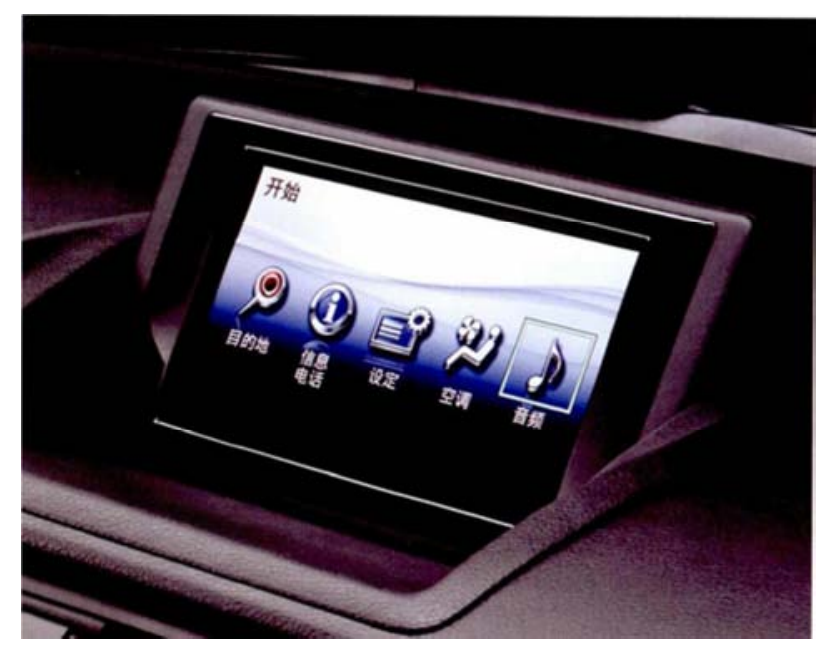

图1 汽车仪表盘服务。

\section{1 . 汽车驾驶安全}

据世界卫生组织的全球道路安全状况报告, 每年约有 数百万人死于道路交通事故, 且全世界每年有上千万人在 道路交通事故中受伤。尽管各项措施都在改善道路安全问
题, 但是汽车驾驶安全研究仍是预防道路伤亡的最重要的 事项之一。在未来的智能网联汽车发展方面, 必须将汽车 驾驶安全问题置于首要考虑问题。

\subsection{1. 司机疲劳, 情绪和压力检测}

实时监测司机状态, 以防止严重的甚至致命的事故, 是未来汽车的基本特征 [2]。通过传感器和计算机视觉应 用程序可以用来确定驾驶员是否疲㤂或者分心, 如利用眼 睛动作或者生物信号处理, 测量闭眼的时间间隔, 检测揉 眼睛和打哈欠，以及司机声音的应力模式; 利用先进的座 椅进行心脏心率变异性监测或转向轮速传感器检测; 通过 呼吸以及触摸传感器检测醉酒状态。一旦确认司机处于危 险状况, 即发出有效警报 (例如由汽车喇叭发出的警报声)。

\subsection{2. 事故避免和协助}

使用安装在汽车上的雷达, 激光和视频传感器 [3], 可以检测即将发生的事故, 并给驾驶员发送信号, 或者汽 车本身可以采取自动决定; 使用车道保持和偏航警告系统, 防止驾驶员偏离正确的轨迹, 借助摄像头探测前方的车道 标线, 同时借助眼睛和头部动态预测驾驶员的意图; 当事 故发生时, 使用智能 SOS 系统自动检测事故的发生并通知 提供道路和医疗援助。

\section{1. 3. 夜视辅助、平视显示器}

先进的增强现实技术可以提高驾驶员在黑暗驾驶条 件下的视觉感知。远红外 (被动) 和近红外 (主动) 是最 常用的夜视增强技术; 将视觉信息 (导航信息, 警告标志, 重大意外危险, 行人过街标志等) 叠加在挡风玻璃上, 可 以最大程度地给予司机正确的信息, 同时最大限度地减少 司机的注意力分散。

\section{1. 4. 远程维护, 车辆援助}

通过远程支援, 汽车信息可以通过网络提供给汽车制 造商, 汽车的有关故障可以提前诊断并排除。在出现事故 后, 通过向驾驶员提供正确的援助以及时解决问题。

\section{2. 智能导航}

目前各大软件公司的路径导航功能已经比较完善, 但 未来智能汽车需要更多的信息交互及人性化的信息呈现。 首先允许用户在家规划路线, 并将其发送给汽车, 并利用 实时信息更新最佳路线，也能提供燃料价格的实时信息 (例如, 周围最便宜的加油站) 和可用的停车地点。其次 导航信息需要呈现在一个触摸模拟平视显示器上, 从而为 驾驶员的主视线提供增强现实信息, 并避免使司机从驾驶 中分心。

\section{3. 能源优化}

目前汽车对能源的利用没有有效的规划, 智能网联汽 车需要采取能够降低、优化能源消耗的技术, 校准汽车功 能, 管理可用的电气能源; 建立电动汽车和智能家庭之间 的接口, 和车到家通信系统, 安排一种车辆有效的充电、 放电时刻表。 


\section{4. 智能娱乐功能}

在汽车行驶过程中, 向驾驶员以及乘客提供娱乐服务, 可以使他们放松从而享受汽车行驶过程。传统的汽车娱乐 功能较为单一, 不能使乘员得到充分的服务。智能网联汽 车可以在这一方面进行加强, 为乘员提供量身定制的更高 级的服务。

\section{4. 1. 智能音乐、视频流媒体}

像现在个人的智能手机或电脑一样, 定制在线流媒体 服务。通过上文情绪感知系统判断司机情绪, 自动选择特 定的汽车行驶环境音乐, 音乐音量和节奏根据情绪自动调 节，以构造一个更舒适、更安全的驾驶体验。同时，提供 这些服务时, 必需考虑不能使驾驶员的注意力从道路上转 移。比如, 视频服务可以考虑安装在后排座位, 只应用于 乘客。

\subsection{2. 道路社交网络}

通过车车通信, 使路上的用户实现社交互动。人们可 以与其他驾驶员分享行程规划的信息, 寻求路上的帮助, 分享经验。将现有的通用社交网络集成到仪表板上, 允许 大多数匿名的邻居信息发送和多媒体信息交换, 以及动态 连接到有相似兴趣、配置或旅行路线的临近用户。

\section{5. 车辆便利性、共享等}

随着人工智能技术的发展, 家居服务和可穿戴设备在 人群中的应用, 智能网联汽车与它们的联系也变得重要起 来。

\section{5. 1. 与智能家居、可穿戴设备的集成}

汽车可以与智能家居功能进行集成, 这样驾驶员在到 家之前就可以启动家用设备, 如照明、取暖、娱乐以及车 库门等。现有的汽车制造商与智能家居环境集成的例子是 福特, 提供了汽车方向盘与亚马逊Alexa的连接, 能访问 其平台上的设备 (如车库门和照明系统)，以及梅赛德斯 奔驰, 允许其驾驶员可以在开车的同时向家里的热量能源 控制系统发送指令。

新型的可穿戴设备与网联车的功能进行集成, 以获取 更加智能和安全的移动性。例如, 福特提供的与车辆集成 的增强视觉眼镜, 同时系统通过智能手表监测驾驶员的血 糖水平和心率。

\subsection{2. 汽车共享}

人口数量的增加, 道路上行驶的汽车也逐渐增多, 造 成更多的道路拥堵和交通事故。汽车共享的出现, 可以降 低人们购买汽车的数量, 出行的需要可由共享汽车得以满 足。而网联车的网络可以使得汽车共享服务更容易扩散传 播, 因为汽车的运动轨迹和车辆状况更容易实时跟踪。

\subsection{3. 驾驶员配置}

网联车应为驾驶员建立配置文件, 为其配置个人设置, 如座椅位置、喜欢的音乐以及驾驶习惯等, 从而提供更好 的驾驶体验。

\section{3. 移动车辆数据}

第二节中讨论了智能网联汽车所需的主要服务, 这些 服务的实现需要汽车数据的支撑, 本节将讨论所需的主要 数据、数据利用和数据涉及的法律问题。

道路上的汽车像分布式传感器网络中的节点, 不断地 收集各种数据。定义这种信息为浮动车数据。最简单的浮 动车数据包含地理数据等。进一步扩展, 可以归结为汽车 的内部数据, 汽车本身或者汽车周围环境数据等。

为了收集内部数据, 数十年来汽车已经配备了大量的 传感器: 多用途摄像头用于监测驾驶员面部或者车道标线; 立体视觉摄像机用于提示驾驶危险; 红外摄像机用于提供 夜间视觉辅助; 雷达和激光探测与测距系统用于生成周围 环境的映射信息; GPS提供地理位置信息; 车轮编码器用 来测量汽车的实际速度; 生物传感器用于观察驾驶员的状 况或者习惯。随着车车通信、汽车与基础设施通信技术的 部署, 不同车辆收集的数据可以在它们之间传输、比较和 集成, 以更好地了解实时道路状况。

安装在汽车或智能手机中的应用程序, 可以获取和充 分利用上述数据。例如, 谷歌数据集维护了大量用于车辆 的数据, 谷歌地图API可以利用地图相关的数据, 并根据 汽车和驾驶员实际情况, 优化导航功能。许多社交网站数 据集也可以作为重要的信息源: 可以收集社交数据(例如, 对朋友最近访问的周围地点或位置的评论) 用于优化驾驶 员的路线，关于附近即将发生的事情的信息也可以获取 $[8]$ 。车车通信所处理的、交换的数据可以成为汽车制造 商非常重要的资产, 并涉及汽车生命周期的每一阶段, 包 括保险公司、租赁公司、应急响应以及其他利益相关方都 能利用这些数据。

汽车收集的大量数据需要上传到云上, 根据一些预测, 在短短一个小时的窗口期内, 一辆汽车可能上传超过 $20 \mathrm{~GB}$ 的信息。为了使得这些数据得到切实有效地处理, 云不仅 要实现数据存储功能, 还必须提供处理和分析能力, 以作 为所有信息的中心枢纽。大量的敏感数据会带来很多的问 题。首先, 要采用大数据分析技术, 区分有用的信息和无 用的信息, 降低管理数据成本。其次, 要解决车载数据的 数据的隐私性问题。2004年, 美国一些著名的汽车制造商 通过了一套隐私和数据安全条例, 主要内容有:

透明度：公司必须告诉顾客他们收集的信息, 以及他 们将在何时何地使用这些信息;

选择: 公司在分享敏感信息或用于营销目的之前需要 征得消费者的同意;

尊重环境: 数据只能在特定背景下被利用或传播;

安全: 技术如果使用得当的话, 会给他们的用户带来 很多优势。但关键数据和隐私数据, 必须得到保护;

数据最小化: 对于数据进行甄别和精简。

\section{4. 通信技术}

智能网联汽车所需的硬件架构包括大量的不同的设 备 (从控制汽车行为的电子元件单元到收集驾驶员输入的 人机界面），通过专用总线连接 [4]。网联车所作的信息 交换能够在几种不同的方式下聚集，下图2 是通信中的一 
部分, 本节列出了一辆智能网联车所需的通信以及所需的 通信技术。

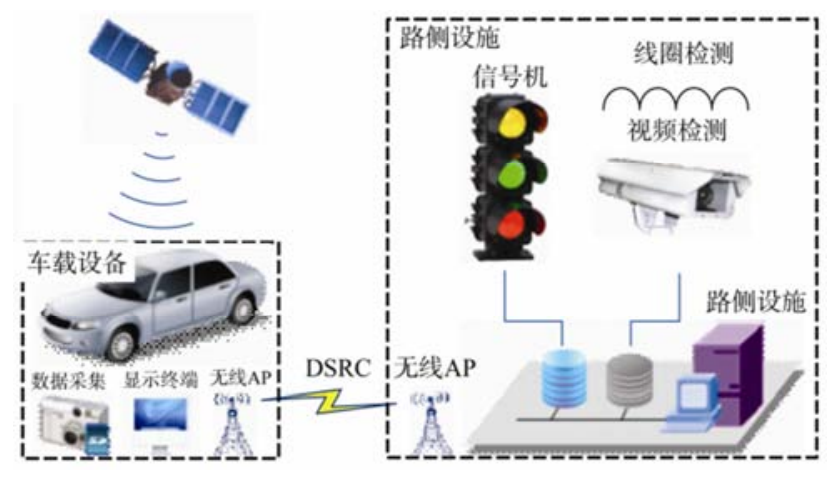

图2 汽车通信。

\section{1. 通信方式}

包含了车辆内部的通信、车与车之间的通信、车与道 路基础设施之间的通信以及车与互联网之间的通信。

\subsection{1. 车辆与车载传感器的通信}

这种通信是汽车电控单元与车内散布的传感器之间 的信息传输, 可以以有线或者无线网络进行。如蓝牙、 ZigBee、UWB, RFID, 或者 $60 \mathrm{GHz}$ 毫米波。需要指出的是, 虽然无线通信减少了车内的布线数量, 但是仍容易出现安 全、可靠性问题。

\section{1. 2. 车车通信}

车辆间的通信是不同车之间的数据传输, 且不需要集 中远程中心的处理。这种通信有助于避免事故、路线优化、 多媒体信息共享 (例如, 事故图片或者附近的停车地点), 以及社交互动。联通汽车形成一个车辆专用互联网 [5]。 已有公司开发车辆行人技术, 通过向行人发送附近危险汽 车的警报以保护行人的安全。

\section{1. 3. 车辆对道路基础设施的通信}

汽车和智能道路基础设施之间的信息交换, 由街道标 志、路边传感器以及交通灯组成 [6]。目的是形成有效的 交通管理，通信一般基于专用短程通信技术。

\subsection{4. 汽车与互联网通信}

智能网联汽车必须能够接入互联网, 体验专用服务和 访问多媒体信息。利用蜂窝网络基础设施将车辆接入互联 网, 使用车辆身份识别模块允许车辆接入 $3 G \backslash 4 G$ 网络 [7]。

\section{2. 几种通信技术}

智能网联汽车的通信中包含两个最基本的问题: 车车、 车与基础设备的通信技术、车与互联网的通信技术。

\subsection{1. 车车通信、车与基础设施通信技术}

常用的通信标准为 IEEE 802.11p标准。2009年，在 车辆连接上IEEE已经开始走向标准化, 创建访问服务的 标准方法以及由不同汽车制造商生产的汽车的交互, 因
此需要进行V2V (Vehicle-to-Vehicle, 即车对车) 和V2R (Vehicle-to-Road Infrastucture, 即车对路) 通信的 整合, 由此促成了 $802.11 \mathrm{p}$ 无线标准的协议的诞生。

系统由安装的路侧单元组成, 如信号灯、道路标志以 及汽车携带的车载单元等。同时, 该系统规定了在移动车 辆环境中必要的架构和服务、数据存储格式、安全信息格 式、支持安全数据交换的网络和传输层服务等等。而且, 该系统支持在智慧交通系统中安全电子支付。

\subsection{2. 车与互联网通信技术}

为了实现网联车的通信, 汽车需要携带类似于手机中 的手机卡一样的SIM卡。利用与 $3 \mathrm{G} / 4 \mathrm{G}$ 网络的连接, 将汽车 连接到互联网上。而关于汽车SIM卡安装在汽车的方案, 提出以下可能方案。

- - 嵌入式解决方案, 将SIM卡永久的装在汽车内, 其应用程序在汽车仪表板上运行。这种技术可在汽车生产 制造时就为汽车安装嵌入式SIM卡, 能够确保每一辆汽车 都具有了通信能力。但是如果需要技术升级或者更换卡时, 可能比较麻烦。

一一系留方案, 汽车安装允许插入个人SIM卡的调制 解调器, 类似于手机的卡槽。这种方案比较简单, 且允许 用户根据技术进步进行升级, 但是需要用户自备SIM卡, 且存在可靠性、安全的问题。

为了实现网联车接入互联网, 汽车必须提供连入互联 网的接口, 以及相关的设置界面。同时, 必须提供对移动 设备无线交互的支持, 以及为用户提供与汽车互动的手段。

\section{5. 自动驾驶汽车}

智能网联汽车的最后一个特征为自主驾驶性。近些年 来, 在汽车市场上讨论最多的话题之一是在未来部署大规 模自动驾驶汽车 (全部或部分) 的可能性。传感技术以及 车载通信技术的发展, 已经为自动驾驶汽车铺平了道路 [9]。一个没有人参与汽车驾驶的概念在一个世纪以来都 是一个工程幻想: 1959年由通用汽车公司研制的火鸟汽车, 能够在高速公路上自主驾驶; 1980年由梅赛德斯公司发布 的一个自主式厢式货车, 利用视觉能力可以在空荡的街道 上行驶；1995年穿越美国项目中，一辆半自主式汽车能够 在人类的帮助下 (仅占了总时间的 $1.8 \%$ ) 行驶 5000 公里; 在2010年VisLab洲际自主挑战赛中中配备了低成本技术 的车辆几乎完全自主的进行了13000公里的旅行, 穿越帕 尔马、意大利到中国上海。

近年来, 许多著名的汽车制造商进行了自主驾驶的探 讨。已有较多数量的谷歌自动驾驶汽车上路了 (已经给予 73 辆汽车牌照)。许多高端汽车上已经 (或者很快将) 应 用了半自动高级驾驶辅助系统, 许多厂商已经透露了他们 自己的全自动汽车原型的概念, 测试报告、开放平台以及 算法已经在科技文献中进行了发表。

本节的目的是列出自动驾驶汽车的基本功能, 并对自 主驾驶汽车的优劣势进行了分析。 


\section{1. 定义和主要功能}

汽车工程师协会给出的对汽车的自动水平的分级, 定 义了大概六级水平的自动驾驶。

0级：驾驶员必须控制每一个驾驶功能;

1级: 这种级别的自动化是指某个特定功能的自动化。 如汽车可以自动转向或加速, 但是总的指挥任务仍由人类 驾驶员来担任, 如车道保持系统等;

2级: 集成了更多的控制功能, 汽车能够真的自主驾 驶。但是, 仍然需要驾驶员监控汽车功能以及周围环境。 驾驶员必须能识别可能存在的危险情况, 并做出反馈应对;

3级: 汽车监控其功能和周围环境, 并且在需要时, 向驾驶员发出接管请求。如, 当计划好的路线结束时, 当 周围环境突变或者和预期的不一样时, 当软件、硬件出现 故障时。

4级: 如果驾驶员无法对接管请求作出反应时, 汽车 能够自主处理紧急状况或者突发状况。

5级: 汽车内完全不再需要驾驶员的存在, 各种操作 和决策都能由汽车来执行。

现在, 大多数汽车制造商正在为汽车提供 1 级功能, 但是一些制造商, 如沃尔沃, 已经在它们的高端汽车上提 供 2 级功能。谷歌计划2018年发布3级自动化汽车。无论达 到哪种程度的自动化水平, 通常来说, 一辆自动化汽车必 须能提供如下所示一组 5 个基本功能:

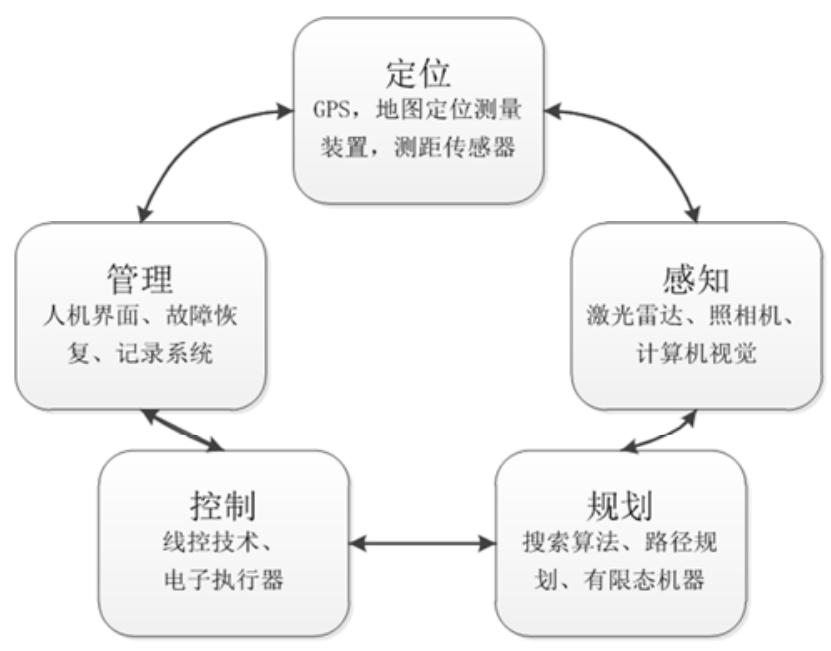

图3 自动驾驶汽车的功能。

定位 [10]: 可以获得任何时刻下车辆的绝对坐标, 并 能够创建周围环境的影像, 获得更精确的道路规划。此项 功能可以通过第二节中的智能导航、驾驶安全服务保障;

感知: 自动驾驶汽车必须能分析周围的环境, 以保持 安全的轨迹, 不与路上的物体或者其他车辆相撞。应鼓励 多传感器的使用, 利用数据融合技术对各种类型传感器收 集的信息进行合并, 以充分利用它们的优势 [11]此项功能 可以通过前面章节中的汽车服务和通信技术支撑, 其中汽 车服务提供感知所需要的数据, 通信技术将数据传输给汽 车。但此外还需要开发数学算法进行数据决策, 常用的数 据决策算法有成分分析法、遗传算法等。它们的基本原理 是建立一套驾驶专用的评估体系, 从所获得的数据提取有
效信息, 并进行评估分析, 得到目标函数的数值, 并由此 给出驾驶指令。如判断汽车是否转弯, 需要综合道路、前 后车、行人位置、行驶速度等信息，不同的信息所在体系 中的重要程度不同, 根据信息给出目标函数值, 并通过函 数值的大小或重要度判断是否可以执行转弯指令。

规划: 必须考虑规划路线中的变量因子, 比如速度、 刹车和转向, 这是基于定位和感知过程中收集的信息, 并 且遵守交通规则。此功能利用搜索算法 (从路径开始到结 束计算最优路线) , 有限状态机 (根据驾驶状态和交通规 则, 选择驾驶行为), 以及路径规划算法（以决定最后的 实际操作) 实现。目前的市场上的规划算法已经比较成熟, 但需要进行更智能的规划, 如第二节中所提到的, 增加更 多的信息交互及人性化的信息呈现;

控制: 线控系统和全电子执行器允许驾驶操作自动执 行。如, 可以利用电子执行器进行转向、加速以及刹车。

管理: 车辆必须收集有关其功能的统计数据, 必须能 够最终进入管理失败模式, 并使驾驶员能够随时获得车辆 控制, 从自动驾驶状态切换到手动模式。这项功能涉及到 管理失败模式的判断, 实质上是一个性能评估的问题, 具 体技术可以与感知功能结合进行, 不同的是管理失败模式 的评估模型更为简单，包括速度失控判断、路线失控判断、 设备失效判断等。

\section{2. 优势与问题}

自动驾驶汽车具有明显的优势, 将为道路安全和交通 管理、机构的财务、个人驾驶员的时间管理和创造性带来 显著地效益, 主要有:

安全: 据世界卫生组织最近的一份报告, 每年由汽车 事故造成的死亡人数多达 125 万人。在没有纠正措施的情 况下, 这个数字预计在未来几年会增长。一般来说, $90 \%$ 的车祸都是由人为失误造成的。很明显, 这种情况随着自 动驾驶汽车的扩散有可能完全避免 [11];

交通优化: 自动驾驶汽车将明显地提高交通状况, 通 过车辆间的持续通信以减少交通拥挤和优化交通流量 [12];

提高能源效率: 交通拥堵和延迟的减少将显著降低排 放与能源消耗。自动驾驶汽车也将比人类驾驶汽车更有效 的燃烧燃料;

新商业模式: 目前, 个人汽车大部分时间都停放和闲 置。自主车辆将使汽车共享更容易和更有效, 无人驾驶车 队可以随时提供载客服务, 小孩, 老人和残疾人, 或者目 前由于任何原因不能驾驶的人们, 都有可能驾车上路。

自动驾驶汽车具有明显的优势, 但同样存在一定的问 题:

花费: 仅仅安装激光雷达系统实际成本为 20 万元至 50 万元, 会导致汽车的价格急剧上升, 成为在市场上扩散的 严重障碍。

技术和标准: 目前的技术和标准仍然处于模型试验阶 段, 距离广泛应用很遥远。应该制定标准来规范, 确保可 靠性;

与非自动汽车的共存: 2016年2月，一辆谷歌汽车发 生了首次由自动驾驶汽车造成的汽车事故。事故原因是由 
自动驾驶汽车没有预测到公共汽车停车而发生碰撞, 人的 意识是很难预测的。但如果要与非自动汽车共存, 就必须 解决这个问题;

用户质疑：顾客可能处于安全性考虑，不想用自主驾 驶汽车取代他们的传统汽车, 而且许多驾驶员享受驾驶的 过程;

失业: 不仅驾驶员, 还有卡车司机、保险公司、交通 协管员, 以及所有其他从事传统交通行业相关的专业人士 将会失业;

责任: 法律制定者应定义明确的规则，在涉及自主汽 车的事故中谁应该负责。

\section{6. 结论}

本文基于国内外智能汽车、智能网联技术等的发展现 状以及国家未来规划情况提出了一个智能网联汽车多方 面发展解决方案。主要从汽车提供的主要服务、所需的通 信技术、自动驾驶的主要功能和优劣势分析三个方面进行 了讲述和探讨。

智能网联汽车的发展已经升级为国家战略问题, 在未 来很长一段时间内都是我国汽车制造业、互联网行业的联 合发展重点。为了在智能网联汽车领域达到国际领先水平, 我国在硬件发展、软件发展、基础设施建设以及规范的法 律法规等方面还需要进一步的完善。

\section{参考文献}

[1] 王兆, 邓湘鸿, 刘地. 中国智能网联汽车标准体系研究 [J]. 汽车智能技术, 2016. 10, 15-18。
[2] 林英姿. $\operatorname{iDRIVE}$ (智能人车交互环境): 我们准备好了吗 [N]. 汽车安全与节能学报, 2016, Vol.7 No. 1, 14-24。

[3] 李克强. 智能网联与未来汽车的技术变革 $[R]$. 科学中国人, 2015. 10, 20-21.

[4] 何长伟, 杨殿阁, 张涛等. 车联网中车载网络负载与线束 优化 $[\mathrm{C}]$. 中国汽车工程学会年会优秀论文, 2014, No. 9, $1-4$.

[5] 黎宇科, 刘宇. 国内智能网联汽车发展现状及建议 $[J]$. 汽车 与配件, 2016 No. 41, 56-59.

[6] 王笑京. 新一代智能交通系统的技术特点和发展建议 [J]. 工程研究-跨学科视野中的工程, 2014. 3, Vol 6 No. 1, $37-42$.

[7] 孟震. 浅谈智能网联汽车通信系统 [Z]. 中国智能交通网, 2016, 102-104

[8] 黎宇科, 刘宇. 国外智能网联汽车发展现状及启示 $[\mathrm{J}]$. 汽车 工业研究, 2016. 10, 30-36.

[9] 远山之石. 智能网联汽车发展综述及浅析 $[\mathrm{J}]$, 上海汽车, 2016. 07, 1-2.

[10] 张懿, 刘焰. 大数据时代下的智能网联汽车发展研究 $[\mathrm{J}]$. 江苏科技信息, 2016. 8, No. 24, 7-9.

[11] 谢志萍, 雷莉萍. 智能网联汽车环境感知技术的发展和研究 现状 $[\mathrm{N}]$, 成都工业学院学报, 2016. 12, Vo; 19 No. 4, 87-92.

[12] 杨晓光, 马万经, 姚佼等. 智慧主动型交通控制系统及实验 [J]. 工程研究-跨学科视野中的工程, 2014.3, Vol. 6 No. $1,43-53$ 。 